\title{
SMLLSTPS: the Software Version of the Macquarie Program, a Computerized Child Assessment System
}

\author{
Erik de Graaf \\ Director, Stichting Down's Syndroom \\ Wanneperveen, the Netherlands
}

\begin{abstract}
This paper describes an application of DataPerfect, which was designed to accommodate the early intervention programme from Macquarie University in Sydney. Not only will its use save time, it can also be used as a powerful tool to monitor the development of children with developmental disability in a way which was not possible before.
\end{abstract}

\section{Introduction: an Early Intervention and a Computer Program}

Present day practice of professional support in the field of early intervention (early, structured support of children with developmental disabilities), carries with it quite some administrative fuss. This is particularly so for professionals, or professional organizations, that support a larger number of children. Just think of the amount of writing involved per child e. g. in producing a detailed activity chart once a week with all the necessary background information. Furthermore, the experiences of the professionals concerned, per task of the program as well as per direction to every single child, have to be administrated, of course, at a central point. This has to be done in such a way that these data are directly available for at least all co-workers of the same organization. Sometimes, particular activities, which are necessary for the assessment of a child, are considered to be so laborious that they are never executed. Drawing up a developmental profile for a differentiated assessment (see below) is an example of that. Finally, a direct comparison between children is not easy with the materials available. The computer program SMLLSTPS (small steps), which is to be presented here, the software-version of the well-known Australian Macquarie Program, is meant to aid in the alleviation of these kinds of problems. As opposed to the Macquarie Program itself, SMLLSTPS is a completely Dutch development.

Before giving a description of the computer program SMLLSTPS some words must be said about the early intervention program named after Macquarie University in Sydney [1]. The version of the Macquarie Program that has been built into SMLLSTPS not only makes use of all five developmental domains that were originally distinguished, but of four others, that were originally presented in a different way $[2,3,4]$, as well. Besides: 
$\mathrm{GM}=$ gross motor

$\mathrm{FM}$ = fine motor / cognitive development

$\mathrm{RL}=$ receptive language

$\mathrm{EL}=$ expressive language and

PS = personal and social skills

the 'new' domains

VS $=$ pre-requisite skills

$\mathrm{RP}=$ reading program

$\mathrm{DP}=$ drawing and pre-writing program

$\mathrm{NP}=$ number skills program

are included as integral components of the Developmental Skills Inventory (or DSI for short) and the Manual of the Macquarie Program. This means that the total number of tasks is no longer 665 but has increased to 786 . The unacquainted reader is referred to the screen prints further on in this paper, to get a feel of the set up of the Macquarie Program.

\section{Hard and Software Requirements}

The computer program SMLLSTPS is an application of DataPerfect. The latter program, as well as its applications, runs on every PC, XT as well as AT and up. However, the production of a number of reports of the application SMLLSTPS, particularly drawing a developmental profile (see below), will take so much time at an XT that moving to an AT might become desirable. But when extensive reports like these are produced at a central place on a faster machine, it is still very well possible to even work with an XT in field.

SMLLSTPS was originally written as an application of version 2.1 of DataPerfect but runs just as well as an application of the so-called Runtime-version of the same program. This last option is much cheaper. However, in that case the support organization of the children concerned cannot adapt the program on its own (although it still can adapt the reports!) and cannot, of course, develop other databases either. One has to think of that before purchasing one of the aforementioned database programs which will enable one to install SMLLSTPS.

Both the aforementioned database programs come from the same WordPerfect'stable'. This means that all kinds of key-stroke combinations, which are known by heart by experienced WordPerfect users, can be applied directly within the DataPerfect environment. E. g. here, too, Shift-F7 means printing and F2 searching, while this program also has to be exited with F7, etc. Another advantage of this close family relationship is the easy transfer of data from DataPerfect to WordPerfect. For example, a developmental profile made with SMLLSTPS (see below) can be imported rightaway into a WordPerfect document.

\section{Personal Data}

Upon starting up the program one enters the panel with personal data (depth 1), called 'NAMECHLD' (name of the child). In this panel every child gets its own record. In addition to his or her personal data it can accommodate data of the respon- 
sible professionals or professional organizations.

By means of a so-called 'door' ('panel link') to depth 2 the panel 'ASSESSMT' (assessment, Screen 1) can be reached. Moving the cursor to that door and pressing arrow-down leads the way to the results of one particular child on the DSI. In this case the results of the child with AI-number 1, which has Down's syndrome, are shown (AI $=$ auto increment). Once in the ASSESSMT panel, the individual results of all children can be recorded there, in conjunction with the remarks which are applicable to a particular child for that particular task. Therefore, this panel is the translation into computer terminology of the right hand part of the pages of the original DSI, notably the columns with pluses and minuses for every single task.

All DataPerfect applications, and therefore also SMLLSTPS, show a type of screen in which the top part is used alternately for the lookup list of the database concerned (see Screen 1) and a help function belonging to that particular application (so-called 'custom help', see Screen 2 below). The bottom part of the screen shows the data within the individual records.

The ASSESSMT panel is shown below in the lookup situation in front of the NAMECHLD panel. In the top part one can see a small portion of the list of 786 tasks of the present version of the Macquarie Program. With the aid of the keys from the cursor block, one can scroll very fast through this list until the required task has been found. In this example that is FM 78 .

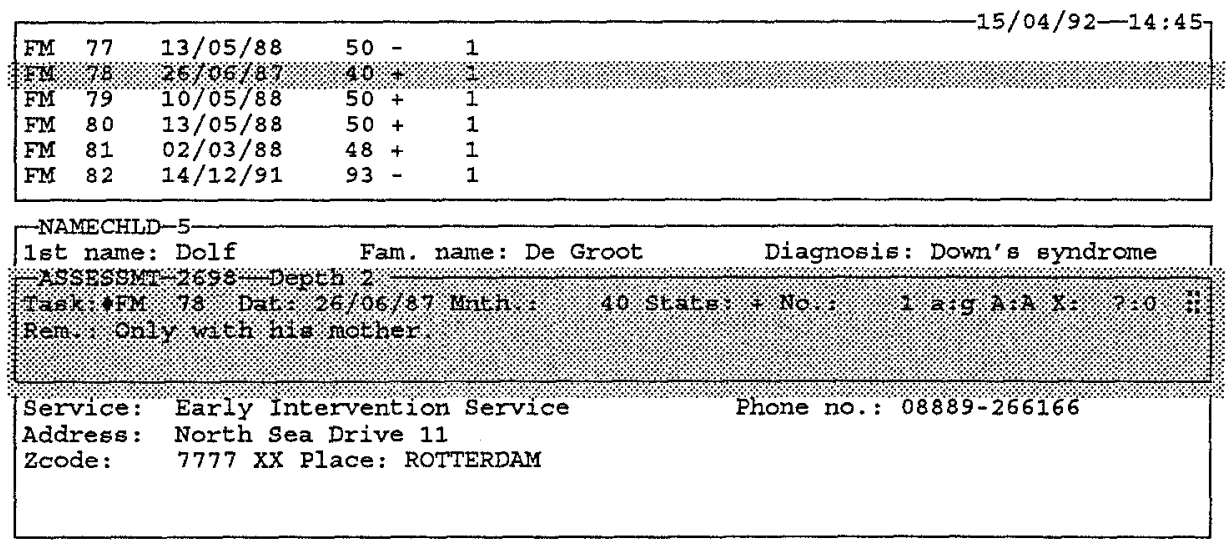

\section{Screen 1}

The field 'Dat' in Screen 1 can accommodate the date of the particular assessment. Via an automatic link with the panel containing personal data, the child's age at that particular date will be included in the field 'Mnth.' immediately. The term 'Stats' stands for the status of the child with respect to that particular task. Three levels are distinguished, notably: ' + ' (yes) for a child that has met the criterion from the Manual of the Macquarie Program, ' \pm ' (nearly) for a child that has almost mastered the task and finally '-' (no) if the child cannot do that particular task. The field 'Rem.' for remarks speaks for itself.

In the lookup list of Screen 1 only the results of child number 1 are shown (see the figures 1 , at the right). Had the door to depth 1 not been opened by arrowdown but by F5 (function key 5) then one would have seen corresponding tasks of 
the various children. In that way, at a glance, one can see how a particular child performs in relation to the others within the database.

While entering data into the ASSESSMT panel, what if you no longer know what is the exact task description belonging to the abbreviation FM 78? Is it still necessary to look into the original books? No, certainly not! For that purpose the field 'Task' in the ASSESSMT panel again forms a door (this time a so-called 'data link'), notably to depth 3, the 'DSI' panel with the descriptions from the DSI of the Macquarie Program (Screen 2). As in all DataPerfect applications, arrow-down means a path to the description of one particular task and F5 a path to all tasks of the DSI. By organizing all available information in this way, task descriptions from the Macquarie Program, which, of course, are equal for all children, are recorded on disk only once. That is a main feature of this application.

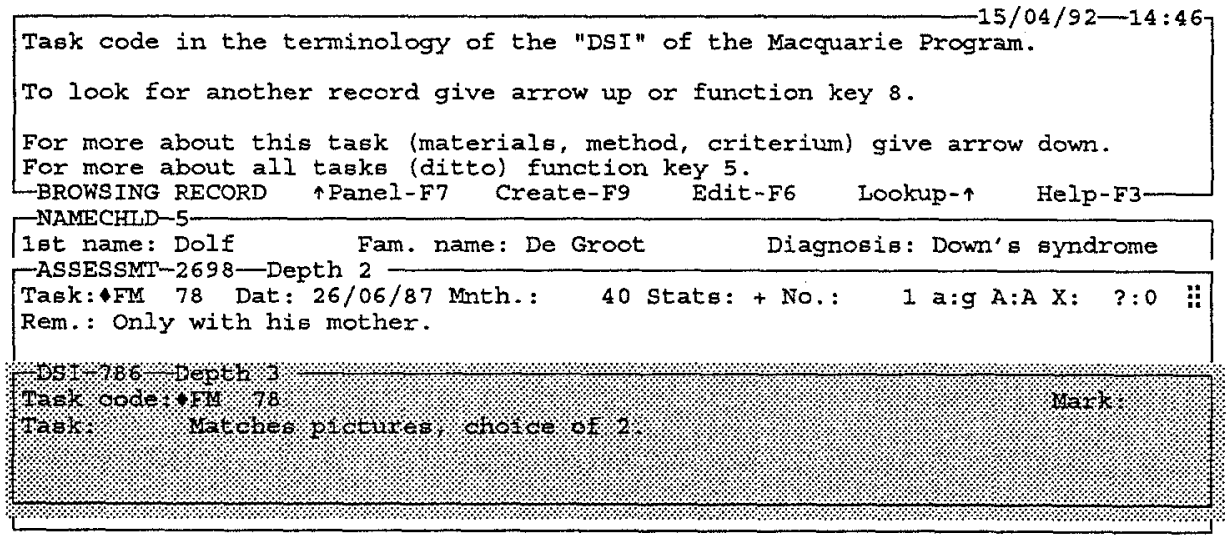

Screen 2

In exactly the same way one can walk from depth 3 , the DSI panel, again via a data link, to depth 4, the 'MANUAL' panel, containing all the extra information belonging to the tasks within the Macquarie Program (Screen 3, next page). As such, it gives the unacquainted reader a good impression of the set-up of the Macquarie Program as well.

Finally, at the extreme right in the MANUAL panel one sees another panel link, this time to depth 5, the 'EXTRAINF' panel, the note book of the professional concerned. In that panel extra information per task, which is relevant for all children, can be administrated. In this way, one can record e. g. references to particular articles, experiences with certain task analyses as well as cross references with other intervention programmes.

The way back to higher levels is found, as always with programmes from the WordPerfect stable, by pressing F7 one or more times. It just depends how far one wants to go back. 


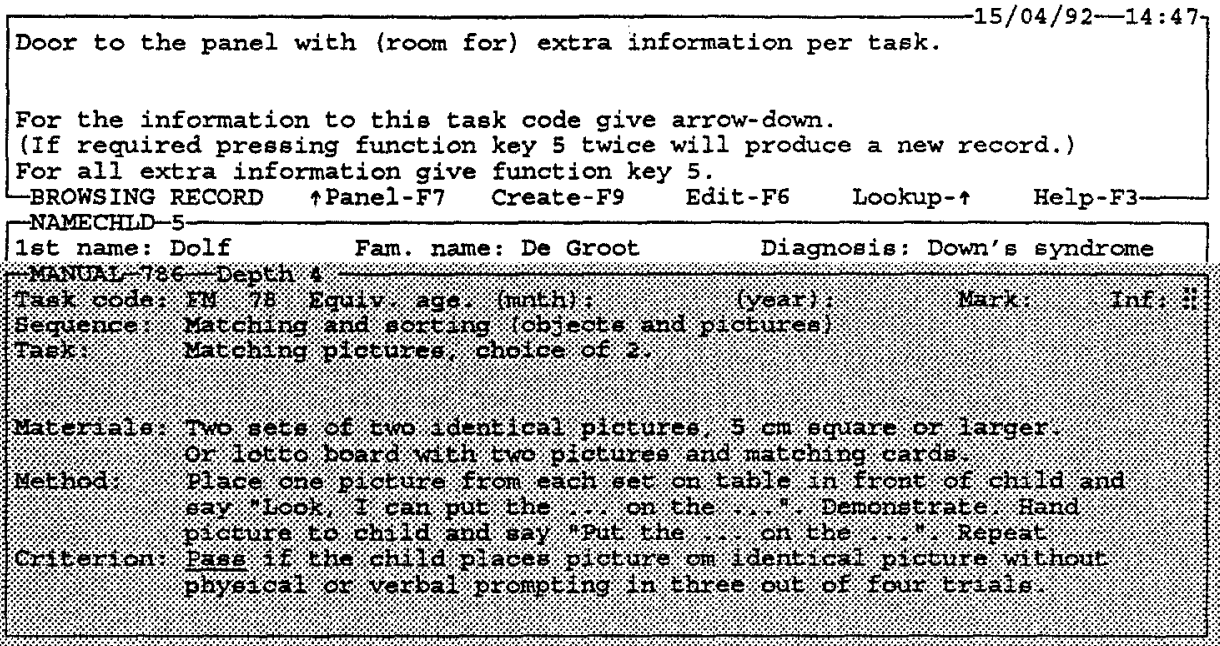

\section{Screen 3}

\section{Reporting}

A very favourable quality of DataPerfect is the enormous range of possibilities to export data from a database, 'reporting'. Within SMLLSTPS extensive use is made of that, too. At the present time the application contains nine standard reports:

1. Built-In Short Reports offers a variety of possibilities to specify all kinds of different reports very quickly, as well as for the production of back-ups and checking of entered data. In addition, data can easily be exported from one computer (teacher, therapist) to another (central place).

2. If large groups of data have to be imported from the DSI of a particular child, which was filled-in by hand, or a pre-existing file has to be updated, this can best be done by using the report Edit a record in ASSESSMT (updating a file). Upon entering the search criteria the computer is able to deal with a whole series of tasks in the desired way in just a few key strokes.

In addition, this option offers the simple possibility of having the same 'correction' automatically entered into the data as often as required. This is needed e. $\mathrm{g}$. at every update for tasks which remain at 'no' at the new date. Furthermore, it might be necessary when data for particular children have to be exported from one computer into another. Clearly, one then has to take into account that two different children in those two computers could, in principle, have received identical AI-numbers. If nothing is done this will most certainly lead to problems. Such an operation can only be carried out without mixing up data by 'correcting' AI-numbers before transferring.

3. In the Personal report tasks are reproduced in such a way that within all nine separate developmental domains first all 'yes' $(+)$ are printed, followed by all nearly's' $( \pm)$ while finally the no's' $(-)$ are given. Within these three groups the tasks are furthermore sequenced according to the date of mastery and finally according to number. In this a way it can be made very clear what that child 'is doing' at that particular time. To avoid this report becoming unreasonably long, particularly with younger children, the report itself 'asks' for the maximum 
number of 'no's' that will be printed. In fact, this report is the equivalent of a completed DSI, including the remarks per task for that particular child.

4. The Personal report of 'no's' and 'nearly's' is a particular case of the previous one. This time tasks for which the child has reached criterion are not reported, but only those tasks that have not been mastered by the child. However, in contradiction to the foregoing report, all available information for the tasks will be printed, also the data from the Manual. As such, this report can be looked upon as a collection of relevant tasks, or rather a plan of work for the intermediate term. There are two different types:

a) a report that is differentiated according to the nine developmental domains from the early intervention programme and

b) a report that is differentiated according to all 29 sequences as a finer subdivision of the developmental domains.

To avoid the reports concerned becoming unreasonably long and parents disappointed because of the number of 'no's' in relation to their child, in both types of report the number of tasks with a 'no' that will be presented can be limited to any specific number entered beforehand. The reports simply 'ask' for its value.

5. The Activity chart contains a description of the 'homework' of one child for the coming intervention period of $\mathrm{e} . \mathrm{g}$. one week. If an $\mathrm{X}$ is placed into field ' $\mathrm{X}$ ' of panel ASSESSMT upon activating this report, the printer will automatically produce the activity chart belonging to that task for that child, with all relevant data.

6. The Mutual comparison of various children shows per DSI-task how long specific children took to reach mastery. In addition an average value is computed.

7. A bit more needs to be said about the Developmental profiles with MDF. The advances in a child's development can be followed exactly on the basis of the results of his or her periodical assessments according to the DSI. That development can further be quantified by calculating the percentage of tasks for which the criterion from the Manual to the DSI has been reached. If a child at an age of e. g. four years shows a ' + ' for about three quarters of all tasks of the DSI and a ' - for the rest then, obviously, that four year old child in that particular domain 'functions on the level of a child of three years' (popular terminology to relate to the concept mental age). That is all the more plausible when one realizes that the child will have mastered the more early tasks in particular, but most probably not the latest ones. By calculating that percentage for every one of the original five developmental domains and by plotting the results, one gets a socalled developmental profile. Upon studying that, one can see at a glance, what the child is good at and also what are the weakest domains that will need most attention. As such, this kind of profile gives a much more differentiated image than the in itself fairly meaningless term 'mental age' without anything more, as is frequently used by professionals. Upon calculating the aforementioned percentages a problem is encountered. Even within the various developmental domains the numbers of tasks per year vary considerably. To accommodate for these differences in numbers one has to use the following formula: 


$$
\sum\left(\frac{\text { number of tasks with } \mathrm{a}+}{\text { total number of tasks }} \times 1 \text { year }\right)_{\text {per year }}
$$

The developmental profiles in this paper are calculated according to this formula. Furthermore, per developmental domain, the report computes the socalled partial fractions: the percentage of tasks scored in relation to the total number in that domain. These partial quotients are of particular interest when one wants to see whether a child gradually starts deviating more or less from the standard after, say, a certain number of months. Next, these quotients are averaged into what has been called the Macquarie Developmental Fraction (MDF). That can be interpreted as a (nonvalidated) measure for development, very much in the sense of IQ. The program does the same calculation again, after exclusion of the language components, to arrive at a non-verbal MDF.

8. Besides the aforementioned report for the production of gross developmental profiles, there is a corresponding report Partial profile GM, FM, RL and PS. Using this, four separate profiles in the developmental domains concerned, which are differentiated into the sequences that make up the domain concerned, are calculated. These last reports in particular can be used very effectively as a diagnostic instrument, for they provide a very accurate image of the development of a child, much more accurate than a test, because of the fact that a developmental quotient is calculated for every separate sequence within every developmental domain.

Both reports prompt the user for the reference date of the desired profiles, so enabling him to produce a report for an earlier age afterwards by simple means, e. g. for a child which is now five for a particular date in its third year of life.

9. In the Personal report in ascending order of tasks, with average time to criterion, all items with a "yes" $(+)$ are presented in chronological ascending order, within all five separate developmental domains. In addition, the report shows the number of months it has taken the child to reach criterion on every particular task. In this way, it immediately becomes clear which tasks in the programme have caused the child concerned particular difficulties.

\section{Further Possibilities}

It is impossible to describe all aspects of SMLLSTPS in full detail here. Therefore, only a very brief description of some other important aspects will be given. Whether the program runs as an application of DataPerfect or of its Runtime-version, for computers with a colour monitor the user can adapt the screen colours himself.

The search function is very important. To produce a report, the child concerned has to be chosen in the NAMECHLD screen. For a report which compares different children one can search for e. g. diagnosis to exclude comparisons of little relevance, e. g. between children with motor limitations due to cerebral palsy and children with Prader-Willi-syndrome.

It is furthermore important to know that the user of SMLLSTPS can easily adapt all reports by himself, or can define his own reports, whether the program runs as an application of DataPerfect or of its Runtime-version. 


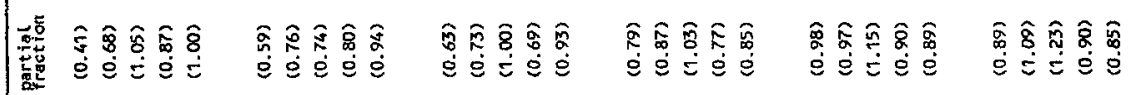

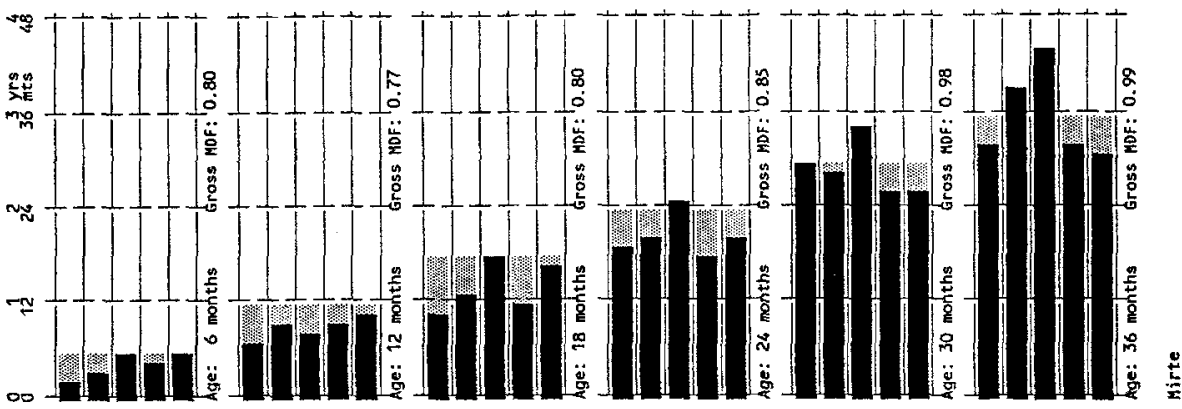

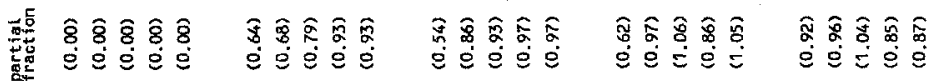

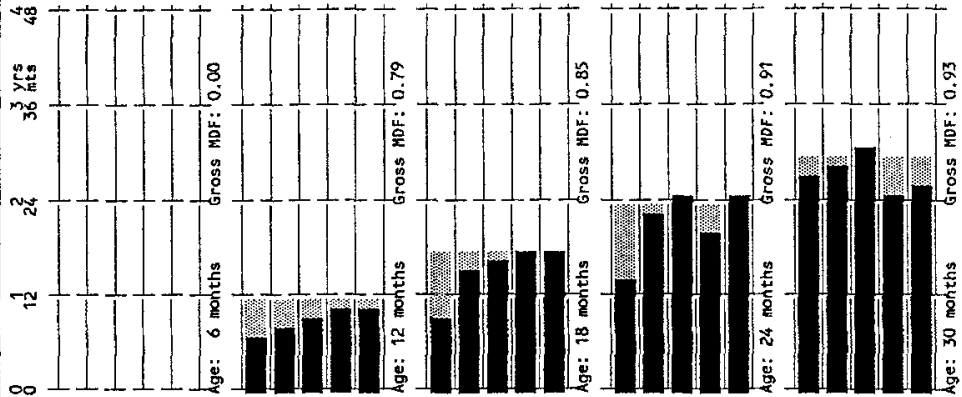

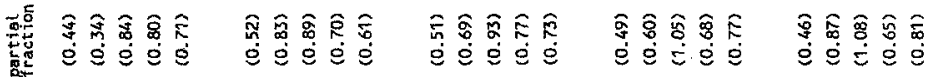

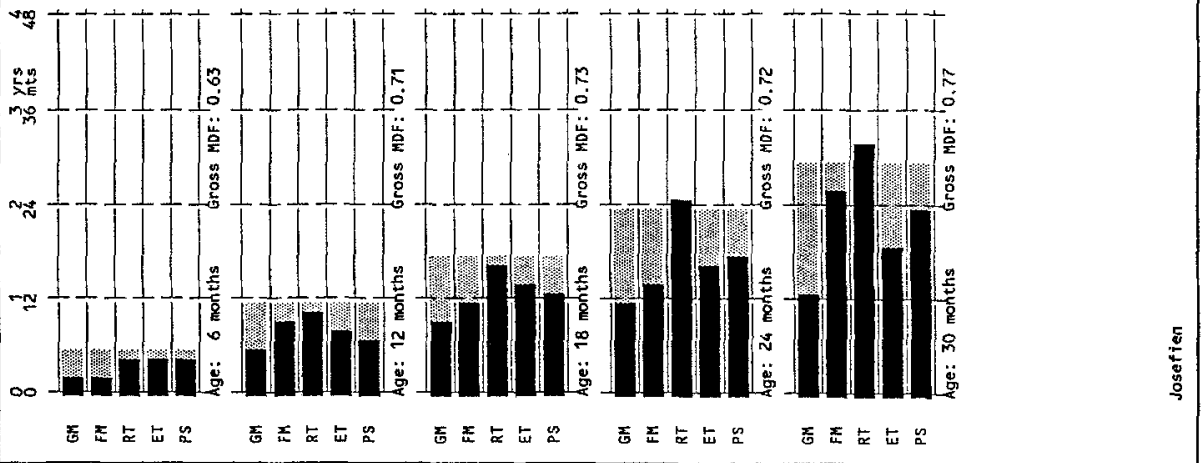


Apart from the application for which the computer programme is offered here, it can also accommodate all kinds of other comparable intervention programmes, or child assessment systems, which work with a checklist (here the 'DSI') on the one hand, and per task in this checklist an amount of additional information (here the Manual) on the other. Programmes like e. g. Portage can be entered into SMLLSTPS to be used subsequently in the way proposed here.

\section{References}

1 M. Pieterse, S. Cairns, and R. Treloar: The Macquarie Program for developmentally delayed children. Manual to be used in conjunction with the Developmental Skills Inventory (DSI). Sydney: Macquarie University, Special Education Centre 1986

2 S. Cairns and M. Pieterse: The Macquarie Program for developmentally delayed children. Reading Program. Sydney: Macquarie University, Special Education Centre 1979

3 M. Pieterse and S. Cairns: The Macquarie Program for developmentally delayed children. Drawing and Prewriting Program. Manual. Sydney: Macquarie University, Special Education Centre 1981

4 Anonymous: The Macquarie Program for developmentally delayed children. Number Skills Program. Sydney: Macquarie University, Special Education Centre 1981

\section{Acknowledgement}

The author wishes to express his thanks to Mrs. Susanne Tonkens-Hart who corrected the English.

\section{Captions for figures on next two pages}

Developmental profiles with MDF for three children with Down's syndrome, notably Josefien, Boy and Mirte, at different ages. The profiles have been produced one by one by SMLLSTPS and were subsequently imported in a WordPerfect text frame.

Partial profiles in the gross motor domain at subsequent age levels for one child with Down's syndrome. The profiles have been produced one by one by SMLLSTPS and were subsequently imported in a WordPerfect text frame. 
Trike Riding

Jumping

Ball skills

Stairs and Climbing

Balancing, Walking, Running

pre-walking skills

Trike Riding

Jumping

Ball skills

Stairs and climbing

Belancing, Walking, Running

Pre-walking skills

Trike Riding

sumping

Ball skills

stairs and Climbing

Batancing, Walking, Running

pre-walking skills

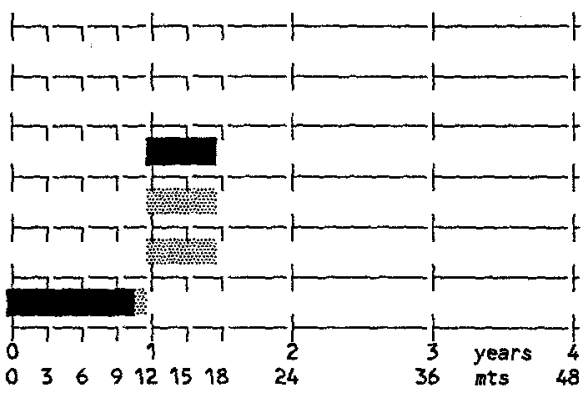

Age: 18 months

sub-partial

fraction

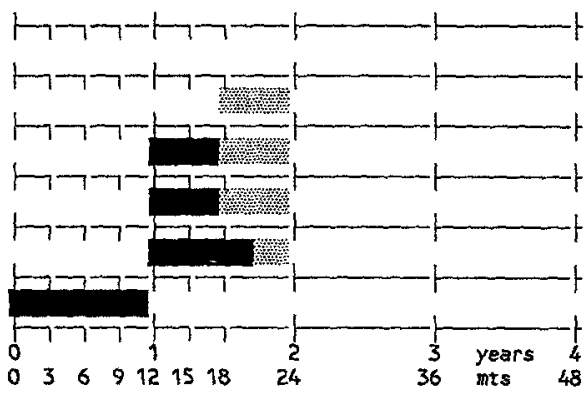

$(0.00)$

$(0 . \infty)$

(0.50)

(0.50)

(0.75)

(1.)

Age: 24 months

sub-partial

fraction

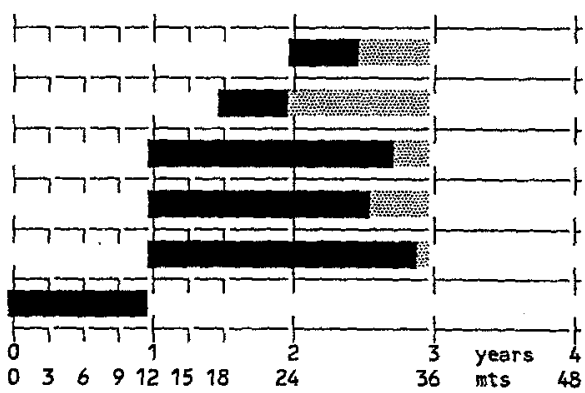

(0.50)

(0.33)

(0.88)

(0.79)

$(0.96)$

(1.00)

Age: 36 months 